Cultures \& Conflits

03 | automne 1991

Mafia, drogue et politique

\title{
Drogue et violence politique au Pérou. Entretien avec Henri Favre
}

Didier Bigo

\section{(2) OpenEdition \\ Journals}

Édition électronique

URL : http://journals.openedition.org/conflits/2017

DOI : 10.4000/conflits.2017

ISSN : 1777-5345

Éditeur :

CCLS - Centre d'études sur les conflits lilberté et sécurité, L'Harmattan

Édition imprimée

Date de publication : 17 octobre 1991

ISSN : 1157-996X

Référence électronique

Didier Bigo, «Drogue et violence politique au Pérou. Entretien avec Henri Favre », Cultures \& Conflits [En ligne], 03 | automne 1991, mis en ligne le 31 mars 2006, consulté le 30 mars 2021. URL : http:// journals.openedition.org/conflits/2017 ; DOI : https://doi.org/10.4000/conflits.2017

Ce document a été généré automatiquement le 30 mars 2021.

Creative Commons License 


\section{Drogue et violence politique au Pérou. Entretien avec Henri Favre}

\section{Didier Bigo} fréquemment. Elle est même au centre d'un discours visant à discréditer le Mouvement révolutionnaire Tupac Amaru (MRTA) mais surtout Sentier lumineux (SL) qui suscite beaucoup plus d'inquiétude et de crainte que son modeste rival. La relation d'ordre symbiotique que cette expression établit entre violence et drogue suggère que les mouvements insurrectionnels opérant dans le pays ne seraient, en définitive, que des bandes de narco-trafiquants encore plus redoutables que les autres, et que les insurgés, loin de poursuivre un objectif politique comme ils le proclament, ne tendraient qu'à des fins crapuleuses. En d'autres termes, la violence serait absolument dépolitisée et totalement criminelle.

4 Paradoxalement, le discours sur le "narco-terrorisme" est contredit de la plus flagrante manière par ceux-là même qui le tienne. Les stratégies que les forces de l'ordre mettent en œuvre contre l'insurrection et le trafic de la drogue reposent sur l'hypothèse qu'il n'y aurait pas de rapport entre les deux phénomènes. L'armée combat le Mouvement révolutionnaire Tupac Amaru et Sentier lumineux sans toucher aux narco-trafiquants ni à leurs intérêts. Et jusqu'à ce qu'un général d'active soit nommé à la tête du ministère de l'Intérieur par le président Fujimori en juillet 1990, la police traquait les narco-trafiquants en évitant de s'attaquer aux insurgés. Elle était d'ailleurs incitée à s'en tenir à cette ligne de conduite par le gouvernement des Etats-Unis qui lui apportait une aide modeste mais appréciée par temps de pénurie. Aujourd'hui encore, les 
autorités américaines veillent à ce que les personnels et les matériels mis à la disposition des Péruviens pour lutter contre le trafic de la drogue ne servent pas à réprimer les mouvements insurrectionnels. Elles craignent non sans raison que leur pays ne soit entraîné à son insu dans un conflit qui changerait alors de nature. Mais pourront-elles longtemps continuer d'agir en fonction d'une perception aussi schizophrène de la situation locale?

C\&C- Quelles sont les pratiques sociales effectives des organisations comme Sentier lumineux ou le Mouvement révolutionnaire Tupac Amaru à l'égard des narcos et quels types de relations entretiennent ces acteurs aux univers sociaux si différents tant dans l'origine sociale, l'idéologie, les motivations?

H. F : Bien qu'elle ait considérablement augmenté au cours des quinze dernières années, la production péruvienne de coca demeure localisée dans les trois zones du versant amazonien des Andes où l'arbuste Erythroxylon coca est traditionnellement cultivé. Ces zones sont, du sud au nord : la vallée de la Convención, dans le département de Cuzco ; la vallée de l'Apurimac, dans le département d'Ayacucho ; et la vallée du Huallaga, dans les départements de Huánuco et de San Martín. De l'une à l'autre, la relation entre drogue et violence varie.

7 La coca produite par la vallée de la Convención continue d'être en partie captée par l'Empresa nacional de coca (ENACO) société à travers laquelle l'État s'efforce d'exercer un monopole de plus en plus fictif- et c'est elle qui satisfait à l'essentiel de la demande licite. Néanmoins, une part croissante de la production échappe à l'ENACO. Elle est clandestinement envoyée hors de la zone où elle sert à l'élaboration du sulfate de cocaïne. La vallée de la Convención, en effet, ne possède pas de véritable industrie de la pâte base. Par ailleurs, son intérêt stratégique est faible. Ni Sentier lumineux ni le Mouvement révolutionnaire Tupac Amaru n'y déploient d'activité militaire.

8 En revanche, la production de coca de la vallée de l'Apurimac alimente sur place une industrie de la pâte base prospère qui est majoritairement entre les mains de Péruviens. Le Mouvement révolutionnaire Tupac Amaru n'a jamais été présent dans cette zone, pas plus que dans le reste du département d'Ayacucho.

Sentier lumineux en a été expulsé par les groupes paysans d'autodéfense que la marine a créés dès 1983 sous le nom de Comités de défense civile, et dont le chef - le fameux "commandant Huayhuaco" - passe pour être un des patrons du cocabusiness local. Cependant, s'ils ne contrôlent plus les producteurs, les sentiéristes contrôlent les intermédiaires qui réceptionnent la pâte base à Ayacucho et qui l'expédient ensuite à Lima d'où elle est acheminée vers les laboratoires colombiens via l'Équateur. Ils perçoivent une sorte de droit d'entrée en ville, tandis que les forces de l'ordre lèveraient l'équivalent d'une taxe de sortie. On voit mal, en effet, comment le sulfate de cocaïne introduit nuitamment dans la cité en état de siège par une myriade de passeurs pourrait la quitter en camions de charge ou en avions de ligne sans la complicité de militaires de tous rangs.

10 C'est cependant dans la vallée du Huallaga que la production de coca et de sulfate de cocaïne a le plus progressé depuis 1975. Estimée alors à moins de 3000 hectares, la surface plantée de coca s'élève à près de 120000 hectares aujourd'hui, et elle continuerait de croître à un rythme supérieur à 10 \% l'an. Le Huallaga est actuellement la première zone cocalera du Pérou et du monde. La quasi-totalité des feuilles récoltées est absorbée par l'industrie de la pâte base que les Colombiens ont installée autour de Tingo María, de Tocache et d'Uchiza, et qu'ils contrôlent encore majoritairement. Le 
sulfate de cocaïne est directement exporté en Colombie par une flotte de petits avions ayant chacun une capacité d'emport de 350 à 500 kilos et disposant d'un réseau de 150 à 180 pistes d'atterrissage ouvertes dans la forêt.

11 Sentier lumineux est intervenu dans la partie supérieure de la vallée du Huallaga dès 1982, amenant le gouvernement à décréter l'état d'urgence dans la zone en 1984. Trois ans plus tard, il relançait son offensive et arrivait à substituer son propre pouvoir à celui de l'État, de sorte que le haut Huallaga devenait une "zone libérée" au sens maoïste de l'expression. En échange d'une garantie d'approvisionnement, les narcotrafiquants se sont vu obligés d'offrir de meilleurs prix aux petits producteurs de coca sur lesquels ils exerçaient souvent des pressions armées. Ils ont également été contraints de désarmer leurs milices et de placer toutes leurs installations sous la protection de l'insurrection. Enfin, ils ont dû accepter de verser l'"impôt révolutionnaire" sous la forme d'une taxe de décollage frappant les avions chargés de pâte base, taxe qui se monterait à 4000 dollars. Trois bandes de narco-trafiquants tentèrent de résister : elles furent impitoyablement massacrées.

Sentier lumineux assure donc la régulation de l'économie de la coca dans le haut Huallaga. Mais, en même temps, il veille à ce que toute la pâte base produite dans la zone soit effectivement exportée à l'étranger, et qu'il n'y en ait point qui s'écoule sur le marché interne. Formellement interdites par l'insurrection à l'intérieur des frontières nationales, la vente et la consommation de drogue sont immédiatement punies de mort. Dans l'"État de Nouvelle Démocratie" que les sentiéristes prétendent instaurer, il y a peut-être place pour les producteurs de pâte base, mais il n'y en a certainement pas pour les dealers ni pour les "camés".

13 On évalue entre 100 et 150 millions de dollars le revenu que Sentier lumineux tire annuellement du cocabusiness dans le seul Huallaga. Reste à savoir ce qu'il fait d'une telle rente qui ne l'a pas amené à modifier sa position doctrinale voulant que les armes ne soient jamais achetées, mais conquises sur l'ennemi. A cet égard, deux hypothèses qui restent à vérifier peuvent être avancées :

14 Sentier lumineux utiliserait l'argent de la coca pour étendre ses réseaux de soutien aux familles des militants et pour développer ses actions d'aide sociale et économique, notamment dans les bidonvilles de Lima, en vue d'élargir son audience dans les milieux paupérisés ; il l'emploierait aussi pour financer le Mouvement révolutionnaire internationaliste, cette cinquième Internationale d'obédience maoïste qui manifeste une surprenante indulgence envers ses membres péruviens dont elle semble loin de partager toutes les opinions.

Quant au Mouvement révolutionnaire Tupac Amaru, il est arrivé dans le moyen Huallaga en 1987, alors que Sentier lumineux était déjà bien implanté dans la partie supérieure de la vallée, avec l'idée de détruire l'industrie de la pâte base et même d'éradiquer la culture de la coca. Cette idée de croisade antidrogue n'a pas tenu longtemps. D'une part, mal formés idéologiquement une partie des militants qui devaient assurer le soutien de la guérilla à partir des villes s'est laissée séduire par les perspectives qu'offrait le cocabusiness. D'autre part, mal préparée militairement, la guérilla n'est jamais parvenue à mettre sérieusement en danger les intérêts des narcotrafiquants, mais elle a constitué une menace suffisante pour conduire ces derniers à resserrer leurs liens avec les forces de l'ordre. Elle n'a pas non plus réussi à contenir Sentier lumineux, qui la rejette de plus en plus au nord, au-delà de Juanjui, vers Rioja, Moyobamba et Tarapoto, et qui vise à soumettre l'ensemble du Huallaga à son pouvoir. 

pour ces acteurs? Les narcos ont semble-t-il besoin d'une base territoriale sûre, et de populations directement sous le contrôle de leurs propres milices armées ; mais, par ailleurs, pour le profit, ils ont tendance à céder sur le contrôle territorial face aux groupes de guérillas. Les réactions des populations locales sont diverses. Comment s'organisent-elles face à ces divers groupes? Quel est le rôle par exemple des syndicats au niveau local, et des municipalités? Y a-t-il allégeance au pouvoir central, à l'État, et refus de coopération avec les guérillas et les narcos ou inversement, n'y a-t-il pas intérêt pour ces populations à accepter un contrôle politique des guérillas, leur garantissant un prix minimum face aux narcos et la possibilité de continuer à cultiver la coca face au gouvernement? géographiques, sociaux et humains communs. L'une et l'autre sont des zones de colonisation agricole récente. Toutes deux ont été peuplées par des migrants descendus des vieilles communautés agraires moribondes des hautes Andes au cours des dernières décennies. Cette population pionnière composée essentiellement de petits et de moyens paysans qui sont propriétaires de leur terre et qui manifestent dans leur comportement un farouche individualisme est naturellement peu réceptive aux messages révolutionnaires, quel que soit leur contenu. Et pourtant, alors que dans l'Apurimac elle résiste victorieusement à Sentier lumineux, dans le Huallaga elle se soumet sans enthousiasme mais de bon gré à l'autorité sentiériste. du Huallaga. Depuis la fin des années 1970, celle-ci est confrontée à une double menace. En premier lieu, le gouvernement, sous la pression des Etats-Unis, relance périodiquement sa lutte contre le trafic de drogue en s'attaquant à la culture de la coca. L'opération Verde Mar en 1979 et la série des opérations Condor au cours de la décennie suivante n'aboutissent peut-être qu'à la destruction de quelques milliers d'hectares de cocales par arrachage des arbustes à la main. Elles n'entretiennent pas moins la paysannerie dans un climat d'insécurité. L'inquiétude - et la colère - des paysans atteint son paroxysme en mars 1989, quand les autorités de Lima font tester près de Tingo Maria un puissant défoliant, le Spike, qui peut être répandu d'avion et qui permettrait d'anéantir en quelques jours d'immenses surfaces plantées en coca. Cette expérience à laquelle une grande publicité est faite, sans doute pour donner le change à l'ambassade américaine, contribue à affermir le pouvoir que Sentier lumineux a acquis dans la zone. Elle provoque, en effet, la mobilisation générale de toute la population derrière les sentiéristes, qui manipulent à leur profit les craintes diffuses que le Spike inspire. Il convient d'observer que toutes les tentatives accomplies par le gouvernement pour réduire la culture de la coca n'ont eu lieu que dans la vallée du Huallaga. Elles n'ont pas affecté l'Apurimac dont on parle d'ailleurs très peu comme d'une zone cocalera.

Les narco-trafiquants colombiens représentent la seconde menace à laquelle la paysannerie du Huallaga doit faire face beaucoup plus directement que la paysannerie de l'Apurimac. Afin d'assurer la régularité de leurs approvisionnements, les narcotrafiquants s'efforcent d'entraîner les petits producteurs de coca dans leur dépendance, et ils n'hésitent pas à recourir à la violence pour contraindre ces derniers à céder leur récolte à des prix arbitraires. L'intervention de Sentier lumineux a désamorcé ce conflit 
qui avait déjà abouti dans certains endroits à l'imposition d'un régime de terreur par les hommes de main des patrons de l'industrie de la pâte.

En prenant en charge la régulation de tout le cocabusiness, sentiéristes ont amélioré les conditions de vente de la coca ; ils ont restauré l'autonomie commerciale des producteurs ; bref, ils ont équilibré le rapport des forces sociales dans la zone. La paysannerie est consciente qu'une défaite de Sentier lumineux risque de la faire retomber sous la coupe des narco-trafiquants alliés ou non avec les forces de l'ordre. C'est pourquoi ils font en sorte qu'elle ne se produise pas, tout en refusant de s'identifier à la cause sentiériste.

21 La tutelle à laquelle Sentier lumineux soumet ses protégés est lourde cependant. A la différence du mouvement révolutionnaire Tupac Amaru qui, dans le moyen Huallaga, cherche à capter l'allégeance des autorités locales, à influencer les organisations syndicales et à canaliser les mouvements populaires, Sentier lumineux impose brutalement son ordre social et moral. Partout, maires, juges de paix et conseillers municipaux sont déchus et éventuellement traduits devant un "tribunal populaire" pour répondre de leurs actes. Les municipalités sont remplacées par des "comités populaires" qui gèrent les services publics (école, dispensaire, etc...), résolvent les différends, font régner sur les mœurs un esprit puritain. Les maisons de prostitution sont fermées, la consommation d'alcool est réglementée, le jeu interdit. Toute activité politique, syndicale ou associative autonome est bien évidemment proscrite. La protection sentiériste se paye de contraintes qui pourraient à la longue engendrer la lassitude.

C\&C- Les populations vivent-elles le fait de cultiver la coca et de la transformer en pâte base de cocaïne sous le mode de la bonne conscience, sous celui de la culpabilité morale, sous celui des impératifs de survie économique, de l'abandon de l'état central et de sa répression incontrôlée... ou selon un mix de ces modalités?

H. F. : Les paysans des Andes cultivent la coca depuis 4 ou 5000 ans, et ils en mâchent la feuille depuis encore plus longtemps peut-être. Dans la civilisation andine, la coca a revêtu de tout temps une singulière importance. Bien de prestige, elle est toujours associée étroitement au pouvoir et à la religion. Elle sert d'étalon d'échange dans les transactions économiques qui se réalisent sur la base du troc. Elle intervient dans le diagnostic de la maladie comme dans le traitement des malades. A ceux qui savent la lire, elle permet de connaître l'avenir. Les spécialistes du surnaturel l'utilisent dans la préparation des offrandes rituelles qui éloignent les esprits mauvais, protègent le troupeau et garantissent la récolte. Tout en contribuant à frayer à l'homme le chemin des dieux, la coca facilite la relation des hommes entre eux. Elle est le lubrifiant social par excellence. Pas de cérémonie, de fête ou de simple réunion familiale, en effet, où elle ne circule pour faire tomber les tensions, oublier les différends et régner la paix. Pas de journée de labeur, non plus, qui ne soit rythmée par des pauses masticatoires à l'occasion desquelles elle vient tromper la faim et effacer la fatigue du travailleur. La bourse contenant les feuilles de coca représente un indispensable accessoire qui complète le vêtement masculin. Parce qu'ils s'inscrivent tous peu ou prou dans la tradition culturelle andine, les producteurs de coca n'éprouvent nul besoin de légitimer leur activité. Il existe toutefois plusieurs versions d'un discours légitimateur de la production de coca qui circulent au Pérou. La version vulgaire de ce discours, celle que popularise Sentier lumineux notamment, joue sur le sentiment national ("notre coca", "la plante sacrée des Incas", "cette richesse nationale que l'étranger veut nous 
empêcher d'exploiter"). Beaucoup mieux articulée, une autre version est construite par les scientifiques sociaux, en particulier les anthropologues. Elle allègue que la coca n'est pas plus responsable de la cocaïnomanie que le raisin n'est responsable de l'alcoolisme ; qu'elle constitue un des symboles identitaires essentiels de l'ethnicité andine ; et que, par conséquent, procéder à son éradication reviendrait à commettre un ethnocide. On trouvera cette version savante exposée dans les trois publications que l'Institut indigéniste interaméricain, agence spécialisée de l'Organisation des États américains, a consacrées à la défense et l'illustration de la coca au cours des dernières années. Seulement les anthropologues oublient peut-être que le "boom" que la coca connaît depuis une quinzaine d'années résulte avant tout de la réponse positive de paysans traditionnels andins à une demande qui n'est ni traditionnelle ni andine. Il est déterminé par le prix auquel les narco-trafiquants consentent à payer la feuille. Bien qu'il ait sensiblement baissé en trois ou quatre ans, ce prix se situe à un niveau très supérieur à celui que peuvent atteindre les autres productions agricoles comme le café, le cacao ou le maïs auxquels les sols du versant amazonien conviennent généralement bien. Il s'ensuit que le paysan est incité à réduire la part de son exploitation qu'il consacre à ces produits et à planter de la coca à la place. L'incitation à la substitution des cultures au profit de la coca est devenue encore plus forte, aujourd'hui que la société publique de commercialisation des denrées agricoles, ENCI, ne remplit plus sa mission. D'une part, à cause de l'état de ses camions - qui ne sont plus réparés - et de l'état des routes - qui ne sont plus entretenues -, ENCI n'est plus en mesure d'enlever toute la production et de l'acheminer vers les grands centres urbains de la côte. D'autre part, en raison de la crise financière qu'elle traverse l'entreprise tend à différer ses paiements. Elle oblige les producteurs à attendre de longs mois un chèque que l'inflation galopante aura amputé de la moitié ou des deux tiers de sa valeur quand il arrivera entre les mains de son destinataire. Ainsi, de plus en plus coupé du marché national, le versant amazonien se convertit à la monoculture de la coca, seul produit qui trouve un débouché local et que l'acheteur paye cher, comptant et en dollars.

24 C\&C- Maintenant que nous comprenons mieux les rapports locaux, peut-on dire quelques mots du rapport à l'État péruvien, tout d'abord sous l'angle des relations de la classe politique péruvienne et des narcos, et ensuite sous celui de la transnationalité? En dehors des zones de production de la coca, a priori, plutôt sous contrôle des guérillas que du gouvernement, existe-t-il des liens avec une "mafia" péruvienne? Que représente, pour le Pérou, l'argent de la drogue? Les zones de production sont-elles intégrées de fait à l'économie des cartels de lu Colombie ou y a-t-il des réinvestissements de l'argent de la drogue dans l'économie légale?

H. F. : A la fin des années 1970, un certain Carlos Lanberg Meléndez, titulaire de divers passeports, entreprend de se hisser au dessus de sa condition de petit patron du cocabusiness. Il y réussit assez bien grâce aux liens qu'il noue à Lima dans certains milieux de la dictature militaire expirante. Incarcéré sous inculpation de trafic de drogue en 1980, il est immédiatement remis en liberté par le ministère de l'Intérieur du général Morales Bermúdez dont il est devenu proche. Il ne contribue pas moins au retour de la démocratie en finançant avec libéralité la campagne électorale de l'Alliance populaire révolutionnaire américaine (APRA) et de son candidat à la présidence, Armando Villanueva. Mais c'est Fernando Belaúnde et l'Action populaire qui l'emportent finalement. Lanberg tombera un peu plus tard, et il ne trouvera personne 
pour le tirer d'affaire cette fois. En 1985, celui qui était peut-être devenu le premier exportateur de sulfate de cocaïne se verra infligé 14 ans de détention.

Sous la présidence de Belaúnde, un autre petit patron de la drogue, Reynaldo Rodríguez López, qui nourrit la même ambition et manifeste le même talent pour l'assouvir, arrive à s'insinuer dans la haute administration. En 1983, il n'est rien moins que conseiller auprès du directeur général de la police. Ses fonctions le mettent en rapport avec les principaux dirigeants politiques et l'aident à entrer dans l'entourage du ministre de l'Intérieur, Luis Percovich, qui prendra Rodríguez avec lui l'année suivante, quand il deviendra chef du gouvernement. L'influence qu'il a acquise et dont il se sert pour étendre ses intérêts économiques lui vaut le surnom de "Parrain". Elle ne l'empêchera pas, cependant, de tomber à son tour. En 1989, le "Parrain" sera condamné à 25 ans de prison. Que d'autres tentatives aient été faites par des narco-trafiquants en vue d'infiltrer la classe politique, c'est possible. Qu'elles aient mieux réussi n'est pas certain. Même si quelques députés ou sénateurs entretiennent des relations plus ou moins étroites avec le cocabusiness, on ne peut parler au Pérou d'un "parti de la drogue" comme il en existe dans certains pays voisins. L'absence de représentation politique du cocabusiness s'explique par la relative faiblesse des narco-trafiquants locaux. Il n'y a pas, en effet, de filière péruvienne de la drogue que ces derniers contrôleraient, mais une filière transnationale à l'intérieur de laquelle ils interviennent en amont, seulement en tant que producteurs de la matière première (la feuille de coca) et fournisseurs du produit semi-fini (la pâte base). Les narco-trafiquants du Pérou sont dépendants des cartels colombiens, qui captent la pâte base, la raffinent dans leurs laboratoires, commercialisent la cocaïne sur les marchés d'Europe et d'Amérique du Nord, et perçoivent l'essentiel des profits. D'autre part, l'industrie de la pâte base, qui était à l'origine entre quelques mains, s'est assez largement déconcentrée. Elle repose sur une technologie relativement simple dont la mise en œuvre ne suppose qu'un modeste investissement. Beaucoup de gros ou de moyens cultivateurs de coca soucieux, d'ajouter de la valeur à leur récolte, ont accédé à cette technologie et produisent euxmêmes la pâte base. La multiplication des petits laboratoires installés sur les lieux de production de la feuille répond également au besoin de réduire le coût du transport afin de faire face à la chute du prix de la cocaïne. Sa conséquence est d'empêcher la cartellisation de la production du sulfate de cocaïne et de rendre difficile l'émergence de grands patrons dans le secteur.

Toutefois, si les narco-trafiquants ne semblent pas avoir l'influence politique qu'on est parfois tenté de leur attribuer, le cocabusiness pèse d'un poids économique qu'aucun gouvernement ne peut se permettre d'ignorer. Il entre pour plus de $60 \%$ dans la composition de la PIB des départements d'Ayacucho, San Martín et Huánuco. Il fournit directement des emplois à plus de 300000 personnes et en fait vivre au moins 2 millions d'autres. Le montant des devises qu'il génère est estimé entre 0,8 et 1,2 milliard de dollars par an, ce qui représente le tiers de la valeur des exportations légales. En abolissant le contrôle des changes, le gouvernement a peut-être moins cédé aux pressions du FMI qu'à la nécessité d'attirer ces coca-dollars sur un marché auquel la Banque centrale de réserve vient actuellement s'approvisionner. Ce marché parallèle, qui constitue un système de blanchiment légal de l'argent de la drogue, fixe les taux de change et exerce ainsi une influence déterminante sur la politique monétaire, financière et économique. En 1567, un haut fonctionnaire espagnol, Juan de 
Matienzo, écrivait que "sans la coca il n'y aurait pas de Pérou". La formule est plus valable aujourd'hui que jamais.

On comprend mieux alors l'ambiguïté de l'attitude gouvernementale envers le cocabusiness. D'une part, le gouvernement doit prendre publiquement position contre les narco-trafiquants et la culture de la coca, et même faire mine de les combattre. Mais d'autre part, il ne peut qu'adopter une attitude tolérante, voire compréhensive, envers un secteur économique dont le pays est devenu si dépendant. En tout cas, aucun gouvernement $\mathrm{n}$ a jamais cherché à empêcher les entreprises publiques Petroperú et Centromín de vendre dans le Huallaga, et à des prix subventionnés, les 60 millions de litres de kérosène et les 35 millions de litres d'acide sulfurique que requiert chaque année l'élaboration de la pâte base.

C\&C- Peut-on dégager quelques tendances, pour les années à venir, concernant la politique gouvernementale, le développement des zones de production...

H. F. : La stratégie antidrogue que les Etats-Unis veulent imposer au Pérou et que les Péruviens font semblant d'accepter est une supply-side strategy. Elle prétend supprimer l'offre de cocaïne en s'attaquant aux narco-trafiquants mais d'abord et surtout aux producteurs de coca. Dans la filière de la drogue, en effet, ceux-ci sont beaucoup plus vulnérables que ceux-là, dans la mesure où ils n'ont pas les moyens d'éviter la détection de leurs cultures, ni de suborner des autorités policières de rang élevé. Mais si les tentatives d'éradication de la coca aboutissaient à des résultats dans certains endroits, la diminution de l'offre de feuilles qui s'ensuivrait provoquerait une augmentation des prix, ce qui inciterait de nouveaux producteurs à entrer sur le marché. La production de feuilles ne serait que délocalisée. Réprimée ici, elle surgirait ailleurs, avec d'autant plus de facilité qu'est grande la disponibilité des terres aptes à la culture de la coca.

Remplacer la coca par d'autres cultures (café ou cacao), c'est-à-dire inverser le sens de la substitution qui s'opère actuellement, ne serait théoriquement possible que si quatre conditions étaient réunies. Il faudrait à la fois : 1 / que le producteur bénéficie d'une assistance technique et financière lui permettant d'élever fortement sa productivité ; 2/ qu'il reçoive $80 \%$ du prix final de sa production, au lieu de $20 \%$ ou moins aujourd'hui ; 3/ que le prix de la feuille de coca reste à son bas niveau de 1989 ; et 4/ que les cours du café et du cacao se maintiennent à leur niveau historique. Autant dire que, aussi longtemps que la demande de cocaïne restera forte dans les pays consommateurs, la coca conservera son énorme avantage comparatif et qu'elle continuera d'être cultivée sur le versant amazonien des Andes péruviennes, même si cette région devait réintégrer l'espace économique et politique national par suite d'une défaite militaire des mouvements insurrectionnels que rien ne laisse au demeurant présager. 
INDEX

Mots-clés : drogue, entretien, mafias et crime organisé, violence

Index géographique : Amérique du Sud 